\title{
O CUIDADO DAS CONDIÇÕES CRÔNICAS NA ATENÇÃO PRIMÁRIA À SAÚDE
}

\section{The care for chronic conditions in primary health care}

\section{Eugênio Vilaça Mendes}

Consultor em Saúde Pública - Brasil

As condições de saúde podem ser caracterizadas como circunstâncias na saúde das pessoas que se apresentam de forma mais ou menos persistentes e exigem respostas sociais reativas ou proativas, episódicas ou contínuas e fragmentadas ou integradas dos sistemas de atenção à saúde, dos profissionais de saúde e dos usuários.

A constatação de que a tipologia clássica de doenças transmissíveis e doenças crônicas não transmissíveis não é capaz de dar suporte à organização dos sistemas de atenção à saúde levou à proposta das condições de saúde, desenvolvida no final dos anos 90 por acadêmicos ligados ao modelo de atenção crônica ${ }^{(1,2)}$ e, depois, acolhida pela Organização Mundial da Saúde ${ }^{(3)}$. Conhecer essas condições permite conhecê-las melhor e, assim, poder prevenir os possíveis agravos através de estratégias de promoção da saúde.

O recorte da tipologia de condições de saúde faz-se a partir da forma como os profissionais, as pessoas usuárias e os sistemas de atenção à saúde se organizam para responder socialmente às demandas colocadas, se de forma reativa, episódica e fragmentada, ou se de forma proativa, contínua e integrada.

Essa tipologia está orientada, principalmente, por algumas variáveis-chaves contidas no conceito de condição de saúde: a primeira relaciona-se ao tempo de duração da condição de saúde, breve ou longo; a segunda, a forma de enfrentamento pelos profissionais de saúde, pelo sistema de atenção à saúde e pelas pessoas usuárias - se episódica, reativa, fragmentada e feita com foco nas doenças e na queixa-conduta, ou, se contínua, proativa, integrada e realizada com foco nas pessoas e nas famílias por meio de cuidados, mais ou menos permanentes, contidos num plano de cuidado elaborado pela equipe de saúde e pelas pessoas usuárias conjuntamente.

É preciso destacar que condição crônica não é igual à doença crônica. Todas as doenças crônicas (diabetes, doenças cardiovasculares, cânceres, doenças respiratórias crônicas, doenças musculoesqueléticas crônicas e outras) são condições crônicas. Mas esse conceito envolve também as doenças infecciosas persistentes (hanseníase, tuberculose, HIV/AIDS, certas hepatites virais e outras), as condições ligadas à maternidade e ao período perinatal (acompanhamento das gestantes e atenção perinatal, às puérperas e aos recém-natos); às condições ligadas à manutenção da saúde por ciclos de vida (puericultura, hebicultura e senicultura); aos distúrbios mentais de longo prazo; às deficiências físicas e estruturais contínuas (amputações, cegueiras, deficiências motoras persistentes e outras); às doenças metabólicas; às doenças bucais não agudizadas; e às condições de saúde caracterizadas como enfermidades (illnesses), que se referem ao modo como as pessoas percebem o seu adoecer, ou seja, a resposta subjetiva do indivíduo e/ou de sua rede de relações frente a uma determinada situação de adoecimento ${ }^{(4,5)}$.

O fracasso do modelo de atenção aos eventos agudos em responder a situações de saúde dominadas por condições crônicas levou, em diferentes países e em diversas instituições, a uma busca por modelos de atenção às condições crônicas. Surgiram, então, vários modelos de atenção às condições crônicas relatados na literatura internacional ${ }^{(6)}$. Os modelos mais expressivos são o modelo de atenção crônica (CCM), o modelo seminal, e o modelo da pirâmide de risco (MPR).

O CCM, proposto nos Estados Unidos da América ${ }^{(2)}$, encontra um ambiente melhor de desenvolvimento em sistemas de atenção à saúde públicos e universais ${ }^{(7)}$, e compõe-se de seis elementos, subdivididos em dois grandes campos: o sistema de atenção à saúde e a comunidade. No sistema de atenção à saúde, as mudanças devem ser feitas na organização da atenção à saúde, no desenho do sistema de prestação de serviços, no suporte às decisões, nos sistemas de informação clínica e no autocuidado apoiado. Na comunidade, as mudanças estão centradas na articulação dos serviços de saúde com os recursos da comunidade. Esses seis elementos apresentam inter-relações que permitem desenvolver pessoas usuárias informadas e ativas, e equipe de saúde preparada e proativa para produzir melhores resultados sanitários e funcionais para a população.

O segundo modelo de grande impacto internacional, o modelo da pirâmide de riscos (MPR), assenta-se na estratificação dos riscos da população. Isto define as estratégias de intervenção em autocuidado e em cuidado profissional. Por sua vez, o cuidado 
profissional, em função dos riscos, define a tecnologia de gestão da clínica a ser utilizada, se gestão da condição de saúde ou se gestão de $\operatorname{caso}^{(8)}$.

As evidências recolhidas na literatura internacional sobre os modelos de atenção à saúde e a singularidade do Sistema Único de Saúde (SUS) fizeram com que se desenvolvesse um modelo de atenção às condições crônicas (MACC) que pudesse ser aplicado ao sistema público de saúde brasileiro ${ }^{(9)}$. A base do MACC é o CCM, mas esse modelo de origem foi expandido, com a incorporação de outros dois modelos, o MPR e o modelo da determinação social da saúde ${ }^{(10)}$, para se adaptar às exigências de um sistema de atenção à saúde público e universal como o SUS.

O MACC está construído em três colunas: numa coluna está a população total estratificada em subpopulações por estratos de risco; noutra coluna estão os diferentes níveis de determinação social da saúde: os determinantes intermediários, proximais e individuais; e na terceira estão os cinco níveis das intervenções de saúde sobre os determinantes e suas populações: intervenções promocionais (nível 1), preventivas (nível 2) e de gestão da clínica sobre as condições crônicas estabelecidas (níveis 3, 4 e 5).

Especificamente em relação à sua aplicação na atenção primária à saúde, fez-se uma pesquisa avaliativa rigorosa no município de Santo Antônio do Monte, Minas Gerais, Brasil. A intervenção ocorreu de junho de 2013 a dezembro de 2014, focando em quatro grupos-alvo: indivíduos com hipertensão arterial, indivíduos com diabetes, mulheres grávidas e crianças menores de dois anos ${ }^{(11)}$. Os resultados, tanto na pesquisa quantitativa quanto na qualitativa, foram muito positivos. Outra avaliação mostrou resultados positivos do MACC na organização em rede da atenção primária à saúde e da atenção ambulatorial especializada ${ }^{(12)}$.

Diante dessa importante temática, o presente número da Revista Brasileira em Promoção da Saúde - RBPS - traz, para complementar esse diálogo, três artigos originais relacionados às condições crônicas em diferentes contextos da Saúde Coletiva e seu impacto na vida dos pacientes. Os principais achados dessas pesquisas dão enfoque: na atividade física como meio de reduzir o risco cardiovascular em idosos hipertensos ${ }^{(13)}$, na associação da deficiência de vitamina $\mathrm{D}$ e suas implicações nos pacientes com diabetes tipo $1^{(14)}$, e por último, os sentimentos de rejeição, tristeza e angústia oriundos de uma gravidez indesejada ao grande impacto na vida psicológica de adolescentes ${ }^{(15)}$.

A RBPS cumpre, assim, uma vez mais, a função de trazer aos pesquisadores e aos leitores temas de grande valia e interesse científico na promoção da saúde.

\section{REFERÊNCIAS}

1. Von Korff M, Gruman J, Schaefer J, Curry SJ, Wagner EH. Collaborative management of chronic illness. Ann Intern Med. 1997;127(12):1097-102.

2. Wagner EH. Chronic disease management: what will take to improve care for chronic illness? Eff Clin Pract 1998;1(1): $2-4$.

3. Organização Mundial da Saúde. Cuidados inovadores para as condições crônicas: componentes estruturais de ação. Brasília: Organização Mundial da Saúde/Organização Pan-Americana da Saúde; 2003.

4. Knauth DR, Oliveira FA, Castro RCL. Antropologia e atenção primária à saúde. In: Duncan BB, Schmidt MI, Giugliani ERJ, Duncan MS, Giugliani C. Medicina ambulatorial: condutas de atenção primária baseadas em evidências. $4^{\mathrm{a}}$ ed. Porto Alegre: Artmed; 2013. p. 66-71.

5. Mendes EV. A construção social da atenção primária à saúde. Brasília: Conselho Nacional de Secretários de Saúde; 2015.

6. Singh D. Transforming chronic care: evidence about improving care for people with long-term conditions. Birmingham: University of Birmingham; 2005.

7. Ham C. Evaluations and impact of disease management programmes. Bonn: Conference of Bonn; 2007.

8. Department of Health (UK). Supporting people with long-term conditions: a NHS and social care model to support local innovation and integration. Leeds: Long Term Conditions Team Primary Care/Department of Health; 2005.

9. Mendes EV. As redes de atenção à saúde. Brasília: Organização Pan-Americana da Saúde; 2011.

10. Dahlgren G, Whitehead M. Policies and strategies to promote social equity in health. Stocolm: Institute for Future Studies; 1991.

11. Andrade MV, Noronha K, Cardoso C, Lorenzi C, Rodrigues CM, Calazans JA et al. Pesquisa de avaliação do laboratório de inovações no cuidado das condições crônicas na atenção primária à saúde de Santo Antônio do Monte. Carta Acordo entre o IPEAD e a OPAS. Belo Horizonte: CEDEPLAR. Universidade Federal de Minas Gerais/ Universidade Federal de São João del Rei; 2016.

12. Huçulak MC, Dalcuche MG. Atenção ambulatorial especializada no Paraná: a experiência da $15^{\text {a }}$ Regional de Saúde. In: Conselho Nacional de Secretários de Saúde. Inovação na atenção ambulatorial especializada. Brasília: CONASS; 2016. p. 82-95. 
13. Hortencio MNS, Silva JKS, Zonta MA, Melo CPA, França CN. Efeitos de exercícios físicos sobre fatores de risco cardiovascular em idosos hipertensos. Rev Bras Promoç Saúde. 2018; 31(2):1-9.

14. Ferraz CLH, Mendes ADN, Ferraz TMBL, Silva CAB. Associação entre vitamina D, controle glicêmico e complicações microvasculares no diabetes tipo 1. Rev Bras Promoç Saúde. 2018; 31(2):1-7.

15. Costa GC, Siqueira DD, Rocha FAA, Costa FBC, Branco JGO. Fatores psicossociais enfrentados por grávidas na fase final da adolescência. Rev Bras Promoç Saúde. 2018; 31(2):1-8.

\section{Endereço para correspondência:}

Eugênio Vilaça Mendes

E-mail: eugenio.bhz@terra.com.br 